\title{
Structural processing of enstatite by ion bombardment
}

\author{
C. Jäger ${ }^{1}$, D. Fabian ${ }^{1}$, F. Schrempel ${ }^{2}$, J. Dorschner ${ }^{1}$, Th. Henning ${ }^{1}$, and W. Wesch ${ }^{2}$ \\ ${ }^{1}$ Friedrich-Schiller-Universität Jena, Astrophysikalisches Institut and Universitäts-Sternwarte (AIU), Schillergäßchen 2-3, \\ 07745 Jena, Germany \\ 2 Friedrich-Schiller-Universität Jena, Institut für Festkörperphysik, Max-Wien-Platz 1, 07743 Jena, Germany
}

Received 29 August 2001 / Accepted 19 December 2002

\begin{abstract}
During their lifetime, cosmic dust silicates suffer from a continuous processing by annealing, cosmic ray and UV irradiation, destruction and possibly also interstellar recondensation. Since the discovery that a significant proportion of stardust silicates leaves their star in crystalline form, the question arose as to why the interstellar silicate dust component does not show any indication of crystallinity. Amorphization due to ion irradiation is one possible explanation for the effect. In this paper, the results of irradiation experiments of submicrometre-sized clinoenstatite $\left(\mathrm{MgSiO}_{3}\right)$ particles with $400 \mathrm{keV} \mathrm{Ar}^{+}$ and $50 \mathrm{keV} \mathrm{He}{ }^{+}$ions are presented. The irradiation doses have been varied between $1 \times 10^{16}$ and $1 \times 10^{18}$ ions $/ \mathrm{cm}^{2}$ for $\mathrm{He}^{+}$ions and $1 \times 10^{14}$ up to $5 \times 10^{14}$ ions $/ \mathrm{cm}^{2}$ for $\mathrm{Ar}^{+}$ions. These doses are comparable to those values that an interstellar silicate grain should be exposed to during its average life-time of $4 \times 10^{8}$ years. Threshold values for amorphization have been amounted to $1 \times 10^{17}$ and $3 \times 10^{14}$ ions $/ \mathrm{cm}^{2}$ for $50 \mathrm{keV} \mathrm{He}{ }^{+}$and $400 \mathrm{keV} \mathrm{Ar}{ }^{+}$ions. Besides the structural changes in the microcrystallites morphological modifications in the grains, but no change of the chemical composition are found. Conclusions of potential astrophysical relevance have been drawn.
\end{abstract}

Key words. ISM: cosmic rays - ISM: dust, extinction - ISM: general

\section{Introduction}

Refractory cosmic dust grains are formed in the outflows of evolved stars. It is generally accepted that silicate grains condense in an amorphous state (Nuth III et al. 1999). When the first ISO SWS (Infrared Space Observatory Short-Wavelength Spectrometer) spectra became available, one of the most surprising discoveries was the finding of clear evidence of the widespread presence of crystalline silicates in envelopes around evolved stars (Waters et al. 1996) and also in Herbig Ae/Be stars (Waelkens et al. 1996; Bouwman et al. 2001). It can be assumed that a portion of the freshly condensed amorphous silicate particles (about 10-15\%) are converted into the crystalline phase by thermal annealing within the circumstellar shell (Molster et al. 1999; Hallenbeck et al. 1998; Fabian et al. 2000). Since the band positions of crystalline pyroxene and olivine are sensitive to the $\mathrm{Mg} / \mathrm{Fe}$ ratio (Cameron \& Papike 1982), the comparison between the spectral signature of synthetic and natural pyroxenes and olivines proves the presence of crystalline $\mathrm{Mg}$-rich silicates that are responsible for the observed sharp MIR and FIR bands in the ISO spectra of circumstellar envelopes (Jäger et al. 1998; Molster et al. 2002a,b).

In contrast, spectra of the interstellar medium (ISM) along the line of sight toward the Galactic Centre show a very broad extinction band at $9.7 \mu \mathrm{m}$ (Lutz et al. 1996; Chiar et al. 2000).

Send offprint requests to: C. Jäger,

e-mail: conny@astro.uni-jena.de
This absorption feature is characteristic of completely amorphous silicates. We have made some simple estimates by superposition of the opacities of amorphous and crystalline silicates, which lead to the result that even $2-3 \%$ of crystalline dust should be noticed in the interstellar silicate absorption spectra. Our estimations have been supported by Li \& Draine (2001) who claimed an upper limit for the content of crystalline silicate grains of any size of less than $5 \%$ on the base of the absence of fine structure in the broad $10 \mu \mathrm{m}$ absorption band. Similarly, the absorption spectra of very young massive stars which are formed by gravitational collapse of molecular cloud cores do not show evidence for the presence of crystalline silicates (Demyk et al. 1999). However, indications for crystalline silicates have been found in young planetary systems of $\beta$ Pictoris type (for review see Dorschner \& Henning 1995; Dorschner 2001).

The absence of crystalline silicates in the diffuse ISM could be the result of very different amorphizing effects: 1 . Amorphization accompanying the shattering processes due to grain-grain collisions, as discussed in the context of life-time and grain-size distributions (Tielens et al. 1987; Jones et al. 1996; Jones 1997). Fast heating and pressure increase within grains can lead to melting and quenching of parts of the dust grains. This fact is experimentally confirmed by the presence of veins or glass droplets in meteorites (Scott et al. 1989) or lunar rocks (Papike et al. 1998). 2. Formation of amorphous silicate particles by recondensation in the diffuse ISM in order to avoid 
the discrepancy between stardust supply and interstellar destruction rates (Jones et al. 1996). 3. Amorphization by heating and quenching processes of dust in the ISM. 4. Amorphization of crystalline silicates by ion irradiation irrespective if the ions come from the low-energy tail of the cosmic rays or from other origins. In this paper we will focus on case 4.

Cosmic rays comprise $\mathrm{H}^{+}, \mathrm{He}^{+},(\mathrm{CNO})^{+}$, and other positive ions with an abundance ratio of about $1: 10^{-1}: 10^{-3}: 10^{-4}$, respectively, and energies between a few $\mathrm{eV}$ and the $\mathrm{GeV}$ range.

Supernova shocks are generally accepted to be the dominant accelerator of cosmic rays up to an energy of about $10^{5} \mathrm{GeV}$ (Biermann et al. 2001). However the source of these ions remains unclear (Lingenfelter et al. 1998). Compared to solar photosphere abundance the cosmic rays are enriched in refractory elements relative to the volatile ones (Meyer et al. 1997). The relatively large abundances of ${ }^{16} \mathrm{O}$, ${ }^{12} \mathrm{C}$ and ${ }^{22} \mathrm{Ne}$ have been expected to stem from Wolf-Rayet $\mathrm{H}$-burning materials suggesting the acceleration of these grains in supernova remnant shocks having velocities between 30 and $6000 \mathrm{~km} \mathrm{~s}^{-1}$ (Maeder 1983; Prantzos et al. 1987; Reynolds 1988; Mewaldt et al. 1996). The directly accelerated dust grains are able to inject these refractory ions by collisions with interstellar gas-phase ions resulting in sputtering and subsequent reacceleration to high energy (Ellison et al. 1997; Lingenfelter et al. 1998).

Ion-irradiation induced modification of solids in space is dominated by protons and helium ions whose intensity is strongest in the low $\mathrm{MeV}$ range. In this paper the term "lowenergy" cosmic rays is used for ions with energies $\leq 10 \mathrm{MeV}$.

The total dose of this low-energy cosmic rays irradiating interstellar silicate dust grains during their life time of about $4 \times 10^{8}$ years is extremely uncertain. The screening effect of the heliosphere and the interaction between solar ions make direct measurements of low-energy cosmic rays impossible, which are representative for the fluxes influencing interstellar dust grains. At energies below $100 \mathrm{MeV} /$ nucleon extrapolation (demodulation) of the measured low-energy flux is model-dependent and consequently measurement errors and demodulation procedure leads to a variation by a factor of about 5 for the proton flux even at relatively high energy of about $1 \mathrm{GeV}$. The uncertainty is rapidly rising (about two orders of magnitude) at a cosmic ray energy of $0.1 \mathrm{GeV}$ (Fields et al. 2001; Mori 1997).

Low energy cosmic ray fluences are very rare in the literature. Since ionization in dense clouds is caused by lowenergy cosmic ray irradiation (Farquhar et al. 1994) the dose of low-energy $\mathrm{MeV}$ protons can be estimated from the hydrogen atom ionization rate. First calculations on the base of the photon production rate due to ionization of hydrogen have been performed by Hayakawa et al. (1961) and were continued in order to account for the heating and ionization of the interstellar gas and grains by Spitzer \& Tomasko (1968) and deJong \& Kamijo (1973). The adopted mono-energetic $\mathrm{MeV}$ proton fluxes were between 1 and $9 \mathrm{H}^{+} \mathrm{cm}^{-2} \mathrm{~s}^{-1}$ corresponding to $10^{17}$ to $10^{18}$ ions $/ \mathrm{cm}^{2}$ assuming a grain life time of $4 \times 10^{8}$ years. Fowler et al. (1970) predicted an upper limit of $2 \times 10^{18}$ protons $\mathrm{cm}^{-2}$ in an energy range between 5 and $30 \mathrm{MeV}$ in order to account for the meteoritic boron isotopic ratio. Morfill et al. (1976) deduced a proton flux between 0.02 and $1 \mathrm{GeV}$ of 1 ion $\mathrm{cm}^{-2} \mathrm{~s}^{-1} \mathrm{st}^{-1}$ $\left(\sim 2 \times 10^{17}\right.$ ions $\left./ \mathrm{cm}^{2}\right)$. Calculations of light isotopes and gammaray line production by low-energy cosmic rays predict a total energy, required to produce the Galactic light isotopes, of $10^{58} \mathrm{ergs}$ (Ramaty et al. 1996). Farquhar et al. (1994) predicted a considerably higher cosmic ray flux in the starburst galaxy M 82 compared to our own Galaxy based on a 700 times higher ionization rate suggesting that indeed supernovae are the major source of cosmic rays. It demonstrates that cosmic ray fluxes can considerably differ dependent on the cosmic environment. One has also to take into account that in the environment of young stars or flare stars the amount of low-energy cosmic rays can be enhanced. Recent direct measurements of proton and helium spectra with different instruments normally starts at energies not much less than $1 \mathrm{GeV}$ (Moskalenko et al. 2002) and provide different results. Assuming an average flux of protons between 20 and $120 \mathrm{GeV}$ a complete flux of about $3 \times 10^{17}$ ions $\mathrm{cm}^{-2} \mathrm{GeV}^{-1}$ can be assumed in the life time of a dust grain.

Interaction between the solid particles and ions may be expected in an energy range of a few eV up to a few MeV. When such an ion penetrates a solid, collisional and scattering processes at the nuclei and the electronic shells lead to an energy loss and a slowing down of the ion, until it will be eventually stopped. However, the alteration of the silicate structure is not well understood so far. Laboratory experiments are necessary to improve the understanding of these processes.

In the astrophysical context, first irradiation experiments of crystalline olivine with $\mathrm{MeV}$ protons and heavy ions were performed by Day (1977) and Krätschmer \& Huffman (1979), respectively. Day (1977) exposed the two minerals olivine and enstatite to protons carrying energies from 1.5 to $2.0 \mathrm{MeV}$ and fluences ranging from $5 \times 10^{15}$ to $7 \times 10^{17}$ ions $/ \mathrm{cm}^{2}$. The author did not measure a change in the infrared spectrum of both minerals indicating that irradiation damage did not occur. Krätschmer \& Huffman (1979) produced a surface layer of highly disordered olivine by exposure of the polished natural mineral to a fluence of $5 \times 10^{16}$ ions $/ \mathrm{cm}^{2}$ of $1.5 \mathrm{MeV} \mathrm{Ne}{ }^{+}$ions.

Amorphization studies of forsterite and five further members of the olivine series had already been performed by Wang \& Ewing (1992) and Wang et al. (1994) for $1.5 \mathrm{MeV}$ krypton ions. The authors demonstrated that the critical amorphization dose increases rapidly with increasing $\mathrm{Mg} / \mathrm{Fe}$ ratio. The differences in the amorphization behavior are probably related to the melting temperature and the bond ionicity in the different olivines. The authors did not report changes in the $\mathrm{Mg} / \mathrm{Si}$ ratio. Bradley (1994) investigated the amorphization process of olivines with $20 \mathrm{keV} \mathrm{H}^{+}$, whereas Dukes (1999) simulated a solar wind bombardment of olivines with $1 \mathrm{keV} \mathrm{H}^{+}$ and $4 \mathrm{keV} \mathrm{He}^{+}$ions. The latter author described chemical changes due to reduction processes of the $\mathrm{Fe}^{2+}$ and a small amount of $\mathrm{Si}^{4+}$ ions to the neutral elements, but no changes of the olivine stoichiometry. In contrast, Bradley (1994) had described the selective sputtering of $\mathrm{Mg}$ and $\mathrm{Si}$ relative to oxygen under an irradiation with $20 \mathrm{keV} \mathrm{H}^{+}$ions. Recent investigations of the amorphization process of the mineral olivine by 4 and $10 \mathrm{keV} \mathrm{He}{ }^{+}$ions were conducted by Demyk et al. (2001). 
They found a depletion of $\mathrm{MgO}$ resulting in the formation of a silicate with a stoichiometry near enstatite even under high fluences. Amorphization studies of enstatite using 1.5 MeV Xe+ ions had been performed (Wang et al. 1998). The authors did not observe a change in the chemical composition and determined the displacements per atom (dpa values) necessary for amorphization with 0.3 .

In this paper, we present new results of irradiation experiments of crystalline, iron-free clinoenstatite, which was found to represent one of the major components of crystalline silicates in evolved stars. It is about four times more abundant than forsterite (Molster et al. 2002a,b). Section 2 describes the experimental procedure, whereas the results of the study are presented in Sect. 3. Section 4 contains a discussion of the astrophysical relevance of the cosmic ray irradiation.

\section{Experimental}

Completely iron-free $\mathrm{Mg}$ silicate minerals are very sparse in nature. Therefore, we synthesized the pure iron-free, crystalline end member $\mathrm{MgSiO}_{3}$ (enstatite) in the laboratory by melting of a mixture of $\mathrm{SiO}_{2}$ and $\mathrm{MgCO}_{3}$ in the corresponding stoichiometric ratio. The melt was held for one hour at $1700{ }^{\circ} \mathrm{C}$ and then slowly cooled down $\left(10^{2} \mathrm{~K} / \mathrm{h}\right)$ to room temperature. It crystallized very rapidly, so that a micro- and nanocrystalline solid was formed. The purity and homogeneity of the produced enstatite was proven by scanning electron microscopy and transmission electron microscopy combined with energy dispersive X-ray (EDX) analysis (Link Oxford) on the nanometer and micrometer scale. Furthermore, the phase purity of the macroscopic amount of the material was confirmed by X-ray diffraction measurements. The synthetic $\mathrm{MgSiO}_{3}$ belongs to the monoclinic crystal system. In the following we use the mineralogical term clinoenstatite. The slight structural differences between the monoclinic and orthorhombic enstatite do not influence the irradiation results. Both materials show the same density, bonding lengths between the metal and oxygen atoms and chain-like structure. The difference between orthoand clinoenstatite is the existence of two symmetrically distinct tetrahedral chains whereby the differences are only very small (Cameron \& Papike 1982). The similarity of both materials has been confirmed by the high coincidence of their IR spectra (Jäger et al. 1998; Koike et al. 2000).

The synthesized sample consisted of nanometer- up to micrometer-sized clinoenstatite particles with irregular shapes. The material was ground in a planetary mill. The particles were dispersed on a TEM grid supported by a lacey carbon film which was directly used for irradiation experiments in a $400 \mathrm{kV}$ implanter, which is able to deliver nearly all elements of the periodic table in an energy range between 20 and $400 \mathrm{keV}$. High energy irradiation experiments were performed at the $3 \mathrm{MV}$ Tandetron accelerator at the Institute of Solid-State Physics in Jena.

$\mathrm{He}^{+}$and $\mathrm{Ar}^{+}$ions with different energies were used for the investigation of the crystalline-to-amorphous transition. The number of displacements per incident ion and unit length $N_{\text {displ }}$, the ratio between the nuclear $\left(S_{\mathrm{N}}\right)$ and electronic energy loss $\left(S_{\mathrm{E}}\right)$ as well as the mean projected range $R_{\mathrm{P}}$
Table 1. Summary of the results of SRIM (Stopping and Range of Ions in Matter) calculations for the $\mathrm{Ar}^{+}$and $\mathrm{He}^{+}$ions used in the irradiation experiments. Additionally, the results of $1.5 \mathrm{MeV} \mathrm{Xe}^{+}$ions are given for the sake of comparison. The values of the nuclear and electronic energy deposition, $S_{\mathrm{N}}$ and $S_{\mathrm{E}}$, respectively, correspond to the maximum of the distribution of the nuclear energy deposition.

\begin{tabular}{crccccc}
\hline \hline Ion & $\begin{array}{r}E \\
{[\mathrm{keV}]}\end{array}$ & $\begin{array}{c}R_{\mathrm{P}} \\
{[\mathrm{nm}]}\end{array}$ & $\begin{array}{c}S_{\mathrm{N}} \\
{\left[\frac{\mathrm{eV}}{\mathrm{nm}}\right]}\end{array}$ & $\begin{array}{c}S_{\mathrm{E}} \\
{\left[\frac{\mathrm{eV}}{\mathrm{nm}}\right]}\end{array}$ & $S_{\mathrm{N}} / S_{\mathrm{E}}$ & $\begin{array}{c}N_{\text {Displ }} \\
{\left[\frac{\text { atoms }}{\text { ionnm }}\right]}\end{array}$ \\
\hline $\mathrm{Xe}$ & 1500 & 380 & 2900 & 2580 & 1.12 & 34.5 \\
$\mathrm{Ar}$ & 400 & 311 & 657 & 810 & 0.81 & 10.5 \\
$\mathrm{He}$ & 50 & 361 & 26 & 58 & 0.45 & 0.57 \\
$\mathrm{He}$ & 400 & 1314 & 22 & 60 & 0.37 & 0.48 \\
$\mathrm{He}$ & 1000 & 2530 & 21 & 72 & 0.29 & 0.47 \\
\hline
\end{tabular}

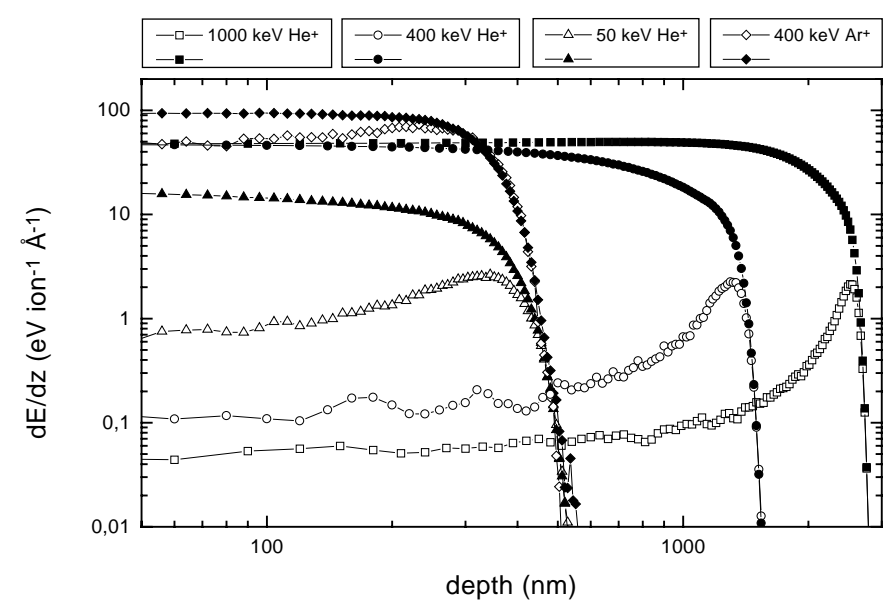

Fig. 1. Comparison of the ratio of the incorporated nuclear and electronic energy loss into the crystalline $\mathrm{MgSiO}_{3}$ target. The solid and open symbols represent the electronic and the nuclear energy deposition, respectively.

were calculated by SRIM 2000 (see Table 1, Ziegler 1996). The target temperature $(70-300 \mathrm{~K})$ and the irradiation doses $\left(1 \times 10^{14}-1 \times 10^{18}\right.$ ions $\left./ \mathrm{cm}^{2}\right)$ were varied in a wide range. In order to compare our results with those of other irradiation experiments, the employed ion fluences $N_{\text {I }}$ were converted into displacements per atom (dpa) according to the equation

$\mathrm{dpa}=N_{\mathrm{displ}} \cdot N_{\mathrm{I}} / N_{0}$,

with $N_{0}$ as the atomic density of crystalline $\mathrm{MgSiO}_{3}$. The current density was held constant at $10 \mu \mathrm{A} \mathrm{cm}^{-2}$ and $0.2 \mu \mathrm{A} \mathrm{cm}^{-2}$ in all experiments for $50 \mathrm{keV} \mathrm{He}^{+}$and $400 \mathrm{keV} \mathrm{Ar}^{+}$, respectively.

The amorphization process was checked by employing transmission electron microscopes (Philips CM120 and the JEOL 3010) in combination with electron diffraction (see Fig. 2). The thresholds of complete amorphization were determined at completely amorphized grains which means that no remains of diffraction patterns were detectable in a range lower than the implantation maximum.

The composition of the grains before and after irradiation was measured by EDX analysis which is attached to the high resolution TEM JEOL 3010. A series of particles with different sizes was selected and electron diffraction patterns before and 


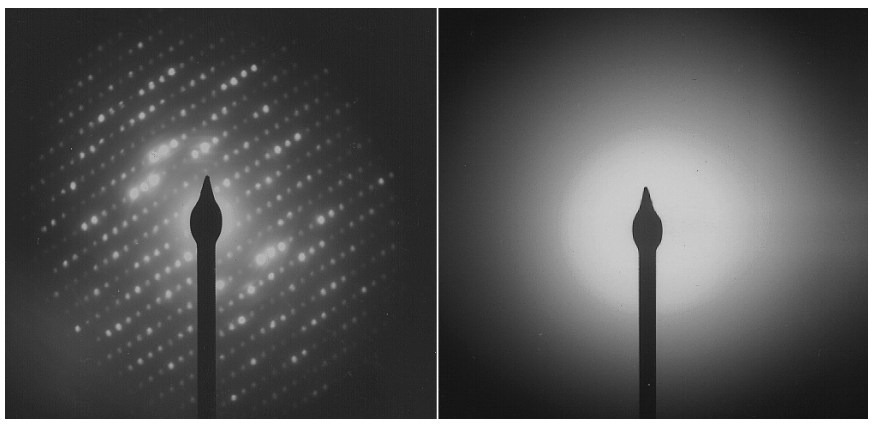

Fig. 2. Electron diffraction image of an enstatite grain before irradiation (left panel) and after complete amorphization (right panel).

after the ion irradiation were used for monitoring the conversion of the structural state.

IR transmission measurements, directly performed at a particle ensemble on the loaded TEM grids before and after irradiation, were recorded with the microscope A590 which is an accessory of the Infrared Fourier Transform Spectrometer IFS 113v. The microscope detector needs to be cooled with liquid nitrogen. Microscope measurements are possible in a wavelength range between 2 and $16 \mu \mathrm{m}$.

\section{Results}

Previous amorphization investigations of enstatite with $1.5 \mathrm{MeV} \mathrm{Xe}^{+}$ions performed by Wang et al. (1998) resulted in an amorphization fluence of $N_{\mathrm{I}, \mathrm{A}}=$ $8.55 \times 10^{13}$ ions $/ \mathrm{cm}^{2}$ for a temperature of $100 \mathrm{~K}$. Table 1 provides the summary of irradiation parameters determined by SRIM calculations for our performed irradiation experiments. We started the amorphization experiments with highly accelerated $\mathrm{He}^{+}$ions $(1 \mathrm{MeV}$ and $400 \mathrm{keV})$ which are closer to the energy distribution of cosmic rays expected in the interstellar medium. Although a similar nuclear energy density (dpa) as in the case of the Xe irradiation was deposited by the $1 \mathrm{MeV} \mathrm{He}^{+}$ions, an amorphization process could not be observed. The examination of the electron diffraction pattern did not provide any hint of partial damage to the crystalline structure.

Amorphization of the clinoenstatite particles was not observed until the He ion energy was reduced to $50 \mathrm{keV}$, but the resulting dpa threshold value necessary for amorphization $\left(N_{\mathrm{I}, \mathrm{A}}=9 \times 10^{16} \mathrm{ions} / \mathrm{cm}^{2}, 5.24 \mathrm{dpa}\right)$ is much higher than that of $1.5 \mathrm{MeV} \mathrm{Xe}^{+}$ions $(0.3 \mathrm{dpa})$. The considered particles have a thickness from about 70-300 nm which is less than the penetration depth of the ions. We always found complete amorphization of the irradiated particles.

In contrast, the amorphization fluence for $400 \mathrm{keV} \mathrm{Ar}^{+}$ions was determined to be about $3 \times 10^{14}$ ions $/ \mathrm{cm}^{2}$, which corresponds to a threshold value of $0.32 \mathrm{dpa}$. The ratio between nuclear and electronic energy loss is much lower for He than for Ar ions and the ratio decreases with higher acceleration energy (see Fig. 1).

Complete amorphization of the crystalline $\mathrm{MgSiO}_{3}$ grains is not only restricted to the region of maximum ion implantation but started at the surface and eventually ends at the
Table 2. Summary of irradiation experiments performed on crystalline clinoenstatite. The term " $\mathrm{iA}$ " in the last column means incomplete amorphization, whereas "A" stands for complete amorphization. The target density used for the calculation of the dpa was $3.259 \mathrm{~g} / \mathrm{cm}^{3}$ corresponding to an atomic density of $9.774 \times 10^{22} \mathrm{~cm}^{-3}$.

\begin{tabular}{ccccc}
\hline \hline & $\begin{array}{c}\text { Dose } \\
{[\text { Ions/cm }} \\
\left.\mathrm{N}_{I}\right]\end{array}$ & $\begin{array}{c}\text { Target } \\
\text { temp. } \\
{[\mathrm{K}]}\end{array}$ & $\begin{array}{c}\text { Displacem. } \\
\text { per atom } \\
\text { dpa }\end{array}$ & $\begin{array}{c}\text { Struct. } \\
\text { changes }\end{array}$ \\
\hline $1 \mathrm{MeV} \mathrm{He}^{+}$ & $1 \times 10^{14}$ & 300 & 0.005 & no \\
& $5 \times 10^{14}$ & 300 & 0.024 & no \\
& $5 \times 10^{15}$ & 70 & 0.240 & no \\
$400 \mathrm{keV} \mathrm{He}^{+}$ & $5 \times 10^{15}$ & 15 & 0.240 & no \\
& $2 \times 10^{16}$ & 15 & 0.962 & no \\
& $1 \times 10^{17}$ & 300 & 4.808 & no \\
$50 \mathrm{keV} \mathrm{He}^{+}$ & $1 \times 10^{16}$ & 70 & 0.583 & no \\
& $5 \times 10^{16}$ & 70 & 2.91 & no \\
& $8 \times 10^{16}$ & 70 & 4.67 & iA \\
& $9 \times 10^{16}$ & 70 & 5.24 & iA \\
& $1 \times 10^{17}$ & 70 & 5.83 & A \\
& $5 \times 10^{17}$ & 70 & 29.16 & A \\
& $1 \times 10^{18}$ & 70 & 58.31 & A \\
\hline $400 \mathrm{keV} \mathrm{Ar}^{+}$ & $1 \times 10^{14}$ & 70 & 0.101 & no \\
& $2 \times 10^{14}$ & 70 & 0.215 & no \\
& $2.5 \times 10^{14}$ & 70 & 0.268 & iA \\
& $3 \times 10^{14}$ & 70 & 0.322 & iA \\
& $3.5 \times 10^{14}$ & 70 & 0.375 & iA \\
& $4 \times 10^{14}$ & 70 & 0.429 & A \\
& $5 \times 10^{14}$ & 300 & 0.537 & A \\
\hline
\end{tabular}

opposite surface. The electron diffraction images, taken at threshold doses, did not show any remains of diffraction patterns. Only in very thick grains, with grain thicknesses larger than the maximum penetration depth, the remaining part of the grain was beyond the implantation range of the ions and therefore not irradiated and not amorphized. However these thick grains were not considered in our experiments.

Of course the determined dpa values calculated by the given equation are based on the numbers of displacements in the maximum of the implantation profiles but taking into account the displacements at about half of the penetration depth the dpa value decrease. Exact values determined are $2.5 \mathrm{dpa}$ and $0.13 \mathrm{dpa}$ for $50 \mathrm{keV} \mathrm{He}^{+}$and $400 \mathrm{keV} \mathrm{Ar}^{+}$ions, respectively, which is about half of the value determined in the maximum. However, the use of the dpa values determined in the maximum of ion penetration (defined calculation procedure within the community of ion irradiation physics) enables us to compare with results of former experiments for example with the results by Wang et al. (1998).

Only ions with a ratio between nuclear and electronic energy deposition $\left(S_{\mathrm{N}} / S_{\mathrm{E}}\right)$ close to 0.4 or more, as in the case of $400 \mathrm{keV} \mathrm{Ar}{ }^{+}$and $1.5 \mathrm{MeV} \mathrm{Xe}^{+}$ions, respectively, are able to amorphize efficiently. A lower ratio of $S_{\mathrm{N}} / S_{\mathrm{E}}$, calculated for low-mass ions like $\mathrm{He}$, requires a rise of the number of displacements, necessary to amorphize clinoenstatite, indicating an in-situ annealing process due to the enhanced ratio between electronic and nuclear energy deposition. 
This result had been previously confirmed for olivines by Wang \& Ewing (1992), who could not trigger an amorphization process by $400 \mathrm{keV} \mathrm{He}^{+}$ions.

However, there is a range of transition into the amorphous state for both, the $\mathrm{He}^{+}$and $\mathrm{Ar}^{+}$ion irradiations, rather than a sharp cut. In the case of $\mathrm{Ar}^{+}$ions the transformation range with incomplete amorphization was found to be between $2.5 \times 10^{14}$ and $3.5 \times 10^{14}$ ions $/ \mathrm{cm}^{2}$. Figure 3 shows two HRTEM images of incomplete amorphization. Remaining lattice fringes of the (011) and (221) planes can be observed. In contrast, remains of lattice fringes of the (001) plane (theory $d=0.49 \mathrm{~nm}$ ) were not found in the high-resolution images. Therefore, the exact value of the critical amorphization dose depends on the orientation of the crystals. Irradiation perpendicular or acuteangled to the (001) surface, which means perpendicular or acute-angled to the $\mathrm{SiO}_{4}$ chains, is more efficient than irradiation of other surfaces. This result is based on the assumption that the susceptibility of pure covalent bonds to amorphization is higher than in the case of higher ionicity in the bond (Naguib $\&$ Kelly 1975). Irradiation, completely parallel to the (001) surface may also lead to channeling effects.

Bradley (1994) reported a significant change in the chemical composition of olivine and enstatite grains after an irradiation with $20 \mathrm{keV}$ protons (total dose: $1.3 \times 10^{18}$ ions $/ \mathrm{cm}^{2}$ ). Similar results had been found by Demyk et al. (2001) under irradiation of the mineral olivine with 4 and $10 \mathrm{keV} \mathrm{He}^{+}$ions. The authors described a selective sputtering and erosion of the exposed surfaces.

Our experiments with $50 \mathrm{keV} \mathrm{He} \mathrm{He}^{+}$ions do not lead to a remarkable sputtering of the grain surface or a change of chemical composition due to a selective sputtering of $\mathrm{Mg}$, even in the case of high fluences, carefully quantified before and after the irradiation by EDX measurements. This is also proven by transmission spectra of the grains measured before and after irradiation in a wavelength range between 7 and $16 \mu \mathrm{m}$ (see Fig. 4). The transmission measurements have been performed on an ensemble of grains at wavelengths between 8 and $16 \mu \mathrm{m}$ by means of the IR microscope.

Crystalline enstatite shows a series of strong bands between 9 and $12 \mu \mathrm{m}$ (see Fig. 4, unirradiated sample). The $10 \mu \mathrm{m}$ band in the irradiated spectra does not shift its center of gravity, which means that the degree of polymerization does not change during irradiation. The spectra simply show the decrease or the loss of the typical enstatite bands. Some remaining signature results from thick grains within the particle ensemble which were not completely amorphized due to the limited penetration depth of the used ions. The degree of polymerization of $\mathrm{SiO}_{4}$ tetrahedra is correlated to the composition of the silicates. A higher content of $\mathrm{SiO}_{2}$ in amorphous $\mathrm{Mg}$ silicate compared to pyroxenes leads to the increase of bridging oxygen and a higher level of polymerization of the $\mathrm{SiO}_{4}$ tetrahedra, which is related to a shift of the $10 \mu \mathrm{m}$ band to shorter wavelengths. Conversely, the reduction of the $\mathrm{SiO}_{2}$ content results in a decrease of bridging oxygen and a shift of the band to longer wavelengths (Mysen et al. 1982). Therefore, the center of the $10 \mu \mathrm{m}$ band provides additional information on the composition of the silicate materials.
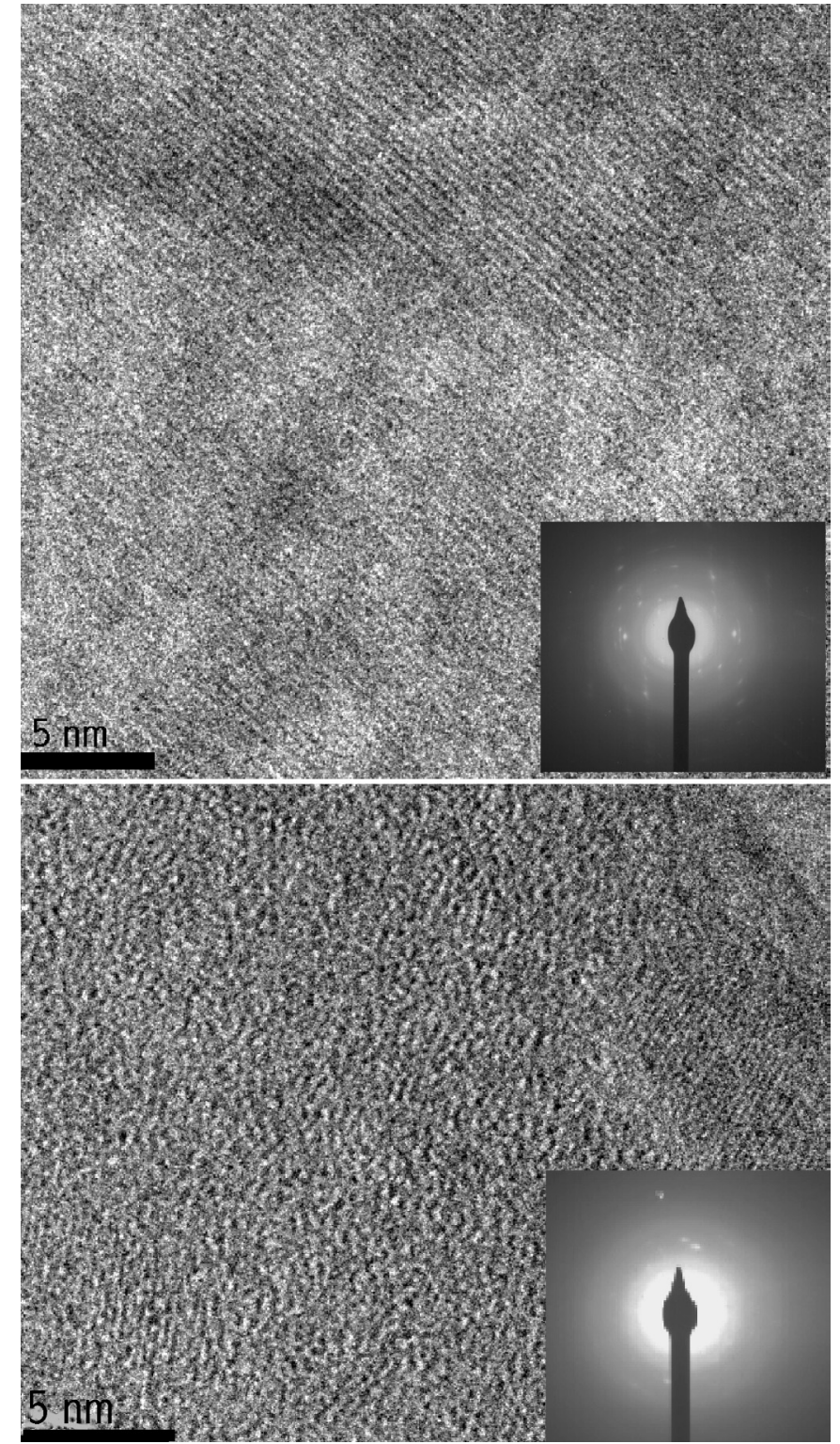

Fig. 3. HRTEM images of two examples of incomplete amorphization. The upper panel shows a larger region of a partly destroyed structure showing the remains of the lattice fringes of the (011) plane with $d=$ $0.44 \mathrm{~nm}$. The lower panel shows two nanocrystals of an average size of 5-8 $\mathrm{nm}$ embedded in an amorphous matrix showing the lattice fringes of the (221) plane $(d=0.305 \mathrm{~nm})$. This kind of incomplete amorphization was found to occur in the clinoenstatite sample irradiated with $400 \mathrm{keV} \mathrm{Ar}{ }^{+}$ions at a fluence of $2.5 \times 10^{14}$ ions $/ \mathrm{cm}^{2}$.

In contrast to the composition, the morphology of the grains can change depending on the ion fluence. Figure 5 shows a clinoenstatite grain before and after irradiation with $5 \times 10^{16}$ ions $/ \mathrm{cm}^{2} 50 \mathrm{keV} \mathrm{He}{ }^{+}$ions. Even before the critical amorphization dose was reached a loss of surface structure and a chamfering of edges and corners of the grains can be observed. This effect becomes stronger with higher ion fluences. Figure 6 demonstrates the morphological change of a group of grains after an irradiation with $1 \times 10^{18}$ ions $/ \mathrm{cm}^{2}$ $50 \mathrm{keV} \mathrm{He}{ }^{+}$ions. The morphological modifications are much more dramatic for fluences higher than the threshold value, and 


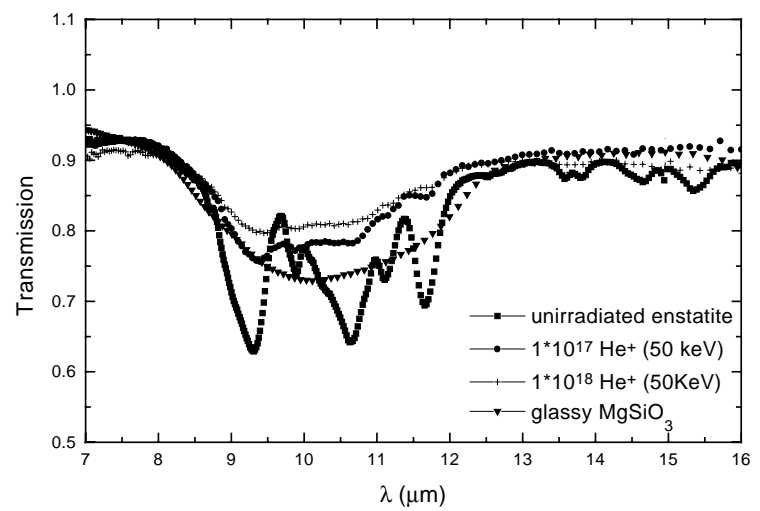

Fig. 4. Transmission spectra of irradiated and unirradiated clinoenstatite compared to a glassy $\mathrm{MgSiO}_{3}$ produced by melting and quenching.

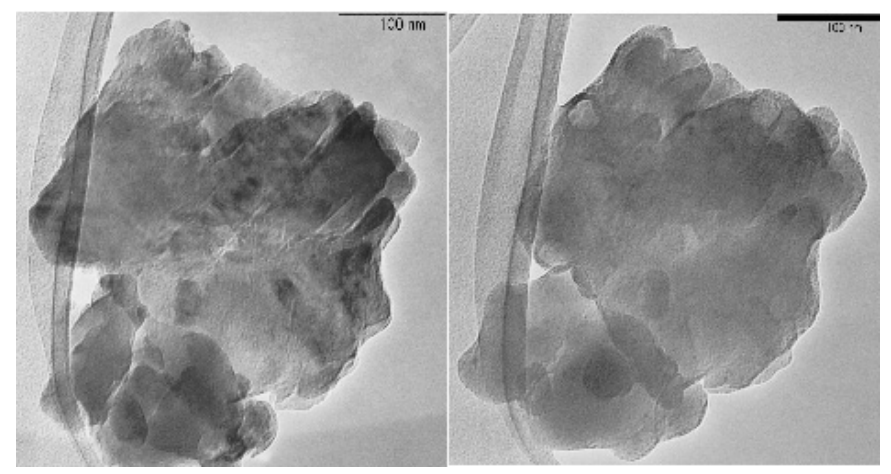

Fig. 5. TEM image of an enstatite grain before and after irradiation with $5 \times 10^{16}$ ions $/ \mathrm{cm}^{2} 50 \mathrm{keV} \mathrm{He}{ }^{+}$ions. It demonstrates the loss of surface structure and the rounding of edges and corners of the grains even before the critical amorphization dose was reached.

they are accompanied by the formation of bubbles or vacuoles in the grains, which had already been found by other authors (see Demyk et al. 2001). The introduced porosity increases with increasing fluence and the size of the pores ranges between 20 and $300 \mathrm{~nm}$. The sizes of the pores are related to the fluence and of course to the grain size. The mean pore sizes in particles ranging from 100 to $200 \mathrm{~nm}$ are between 20 and $40 \mathrm{~nm}$. Extremely large pore sizes as can be seen in Fig. 6 are only formed in larger grains (note that the grains in the given example have an average diameter of $500 \mathrm{~nm}$ but a lesser thickness). At a fluence of $1 \times 10^{18}$ ions $/ \mathrm{cm}^{2}$ the degree of porosity is about $50-60 \%$. The formation of bubbles seems to be related to a short, local heating of parts of the grains above the glass transition temperature within the collision cascades. In this temperature range the solubility of gases previously dissolved in the enstatite grains decreases and gases can be released. By sufficient cooling of the material the formed porous structure cannot relax and remains conserved, whereas a part of the released gas can redissolve again into the structure or leave the material through open pores. The formation of He bubbles can be excluded since the calculated penetration depth is in the range of $360 \mathrm{~nm}$ and the formation of bubbles was also observed in very thin grains $(\leq 150 \mathrm{~nm})$, where He had passed the grains completely.

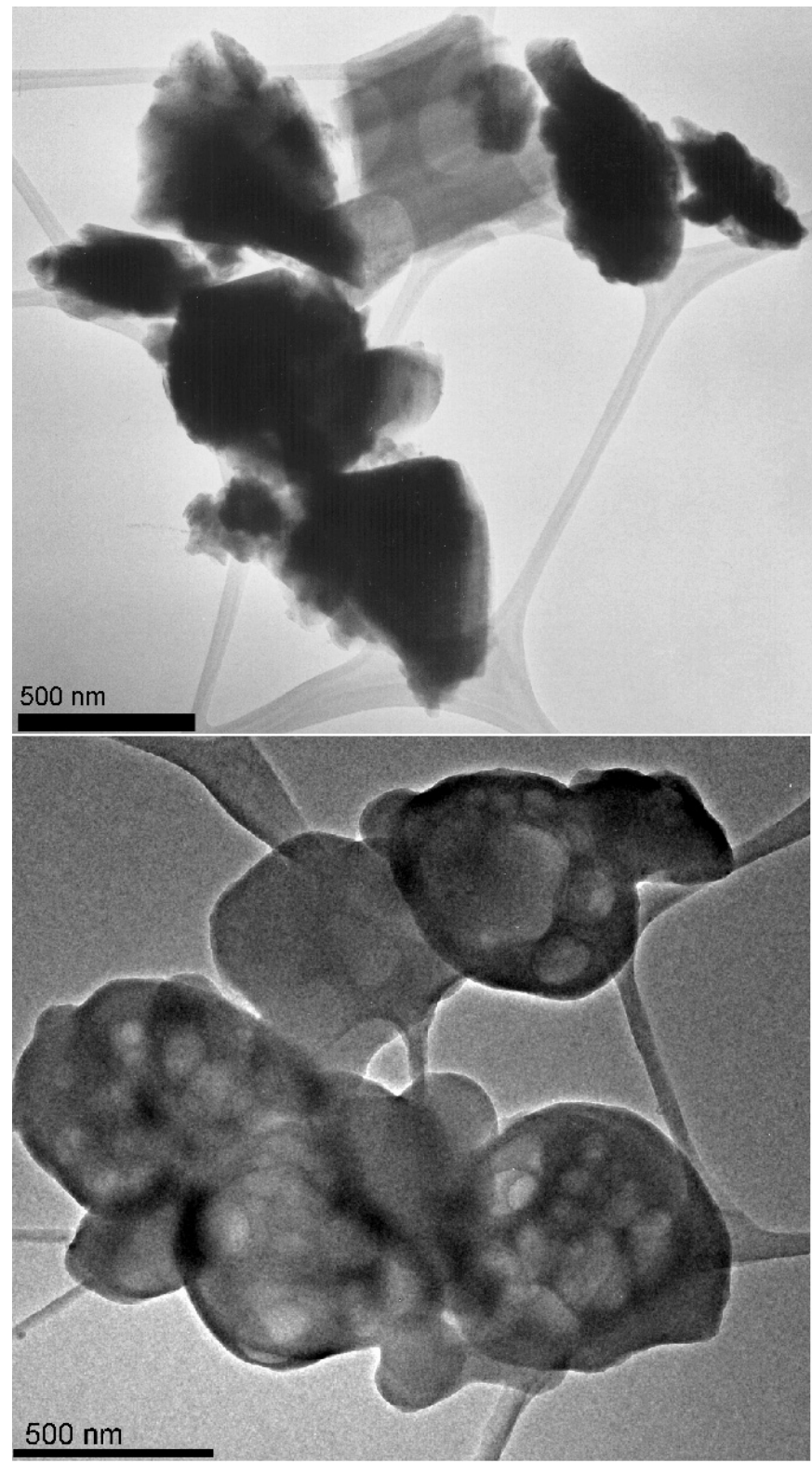

Fig. 6. TEM image of a group of grains before (upper panel) and after an irradiation with $1 \times 10^{18}$ ions $/ \mathrm{cm}^{2} 50 \mathrm{keV} \mathrm{He} \mathrm{H}^{+}$ions (lower panel, rotated counter-clockwise by about $45^{\circ}$ ). Please note the dramatic morphological transformation of the grains.

\section{Conclusions of astrophysical relevance}

While high-energetic cosmic rays $(E \gg 1 \mathrm{MeV})$ pass the interstellar dust grains without substantially changing the internal material structure, our experiments with $\mathrm{He}^{+}$ions of $50 \mathrm{keV}$ and $\mathrm{Ar}^{+}$ions of $400 \mathrm{keV}$ confirm that ions of this energy range can effectively amorphize the interstellar low-temperature silicate grains. For our experiments, we used synthetical clinoenstatite grains of about the same size (in the order of $0.1 \mu \mathrm{m}$ ) as the silicate grains in interstellar space. This monoclinic variety of enstatite was also used as laboratory analog (Jäger et al. 1998) for the identification of crystalline silicates in ISO-SWS spectra of evolved stars. In these sources, enstatite $\left(\mathrm{MgSiO}_{3}\right)$ 
was more abundant than forsterite by a factor 3-4 (Jäger et al. 1998; Molster et al. 2002a,b).

According to our experiments, the irradiation doses necessary for total amorphization of submicrometre-sized grains amounted to $1 \times 10^{17} \mathrm{~cm}^{-2}$ for $\mathrm{He}^{+}$ions and $3 \times 10^{14} \mathrm{~cm}^{-2}$ for $\mathrm{Ar}^{+}$ions. Since the light ions are much more abundant than heavier ones, we consider our results with $\mathrm{He}^{+}$ions rather representative for the interstellar amorphization problem than those of the $\mathrm{Ar}^{+}$ions. However, one has to keep in mind that the less abundant heavier ions are much more effective in amorphization.

Demyk et al. (2001) have shown that effective amorphization of crystalline olivine can already be triggered by $\mathrm{He}^{+}$ions accelerated in shock waves with velocities from 400 to $700 \mathrm{~km} \mathrm{~s}^{-1}$ corresponding to energies $4-10 \mathrm{keV}$. However, this amorphization is accompanied by selective sputtering processes. Our experiments indicate that $\mathrm{He}^{+}$ions at $50 \mathrm{keV}$ coming either from the low-energy cosmic rays or from fast shock waves with velocities of about $1500 \mathrm{~km} \mathrm{~s}^{-1}$ do amorphize the grains without sputtering. Since the fluences and the energy distribution of both ion sources are still uncertain we are not able to quantitatively charge up their effectiveness.

Since the sizes and the composition of our experimental particles are comparable to those of the interstellar silicate particles we think that our results can give a first orientation on the interstellar amorphization process if the total irradiation dose irrespective of their origin and acceleration mechanism in the interstellar environment during the mean life-time of an average silicate particle $\left(4 \times 10^{8}\right.$ years, Jones et al. 1996) is in the same order than our experimental dose.

Ion irradiation experiments simulating the dust processing and sputtering in different astronomical environments (Strazzulla et al. 1983; Strazzulla \& Baratta 1982; Léger et al. 1985; Foti 1991; Jenniskens et al. 1993; Mennella et al. 1997; Duley 2000) are based on the assumption that the astrophysical relevant dose of low-energy ions is between 3 and 10 ions $\mathrm{cm}^{-2} \mathrm{~s}^{-1}$ corresponding to fluences of $1 \times 10^{17} \mathrm{~cm}^{-2}$ to $1 \times 10^{18} \mathrm{~cm}^{-2}$, which is quite similar to our experimental doses for $\mathrm{He}^{+}$.

Certainly, the relevance of dust amorphization by ion irradiation depends on the possibility of dust annealing processes which could trigger a recrystallization process. Stochastic annealing of the interstellar dust grains by starlight absorption, cosmic ray heating or heating by shock waves can be accounted for direct competition to the amorphization process caused by ion irradiation.

Steady-state temperatures of silicate dust grains in the interstellar medium subjected to grain size and shape have been determined to amount between 12 and $15 \mathrm{~K}$ (Voshchinikov et al. 1999).

Heating of dust in shock waves has been discussed by Dwek \& Werner (1981), Itoh (1988) and Dwek et al. (1996). The large relative motion between the dust and gas can lead to an increase of temperature by ion impact. The grain heating depends on the shock wave velocity, grain size and density and was amounted to a maximum value of $150 \mathrm{~K}$ for a preschock density of $n_{\mathrm{o}}=1 \mathrm{~cm}^{-3}$ and a gas temperature of $10^{6} \mathrm{~K}$. An increase of the density to $100 \mathrm{~cm}^{-3}$ leads to a maximum temperature of about $350 \mathrm{~K}$.

Draine \& Li (2001) have calculated the grain heating by starlight for differently sized and composed grains which was found to be most efficient for ultrasmall grains. For example, a silicate grain of a few tenth of nm reaches an upper temperature limit of $700 \mathrm{~K}$ whereas grains of about $1.5 \mathrm{~nm}$ attain temperatures less than $100 \mathrm{~K}$ even at absorption of UV light. Radiative cooling times are, of course, size-dependent and were amounted to 0.1 and about $5 \mathrm{~s}$, respectively. The lack of a $10 \mu \mathrm{m}$ emission band and the run of the UV curve at about $6.5 \mu \mathrm{m}^{-1}$ set an upper limit of less than $10 \%$ of grains smaller than $1.5 \mathrm{~nm}$ (Li \& Draine 2001).

Cosmic ray heating of grains, a relevant process in dense molecular clouds, has been investigated by different authors (Léger et al. 1985; Hasegawa \& Herbst 1993). Following Léger et al. (1985) and Hasegawa \& Herbst (1993) a peak temperature of $70 \mathrm{~K}$ can be achieved.

However, annealing experiments of our amorphized enstatite samples up to $880 \mathrm{~K}$ have demonstrated that spontaneous recrystallization of the amorphous grains does not occur. Therefore we can conclude that the discussed annealing processes are not efficient enough to trigger recrystallization but may have repercussions on processing or evaporation of ice mantles around the grains.

Wang \& Ewing (1992) had proven that the critical dose necessary for amorphization of olivines rapidly increases with increasing magnesium content. The critical amorphization dose for fayalite is less than $1 / 4$ of the critical dose of $\left(\mathrm{Mg}_{0.88} \mathrm{Fe}_{0.12}\right)_{2} \mathrm{SiO}_{4}$ for $1.5 \mathrm{MeV} \mathrm{Kr}^{+}$ions at room temperature. Wang et al. (1994) could demonstrate that the dpa value necessary for amorphization of forsterite increases exponentially beyond temperatures of about $650 \mathrm{~K}$. These results could account for the fact that iron-containing silicates in evolved stars, protoplanetary nebulae and young stellar objects are found to exist in the amorphous state (Molster et al. 2002a,b). A continuous irradiation of the silicates by ions at elevated temperature of the respective solar or galactic origin could avoid the crystallization of this material.

A second result of astrophysical relevance is that we did not find any indications in our experiments that in the energy range of the ions the composition of the target particles was changed. This result finds support by earlier experiments (Wang et al. 1998; Wang \& Ewing 1992; Dukes et al. 1999). Apparently, such chemical changes are very sensitive to the energy range, as the experiments by Demyk et al. (2001) and Bradley (1994) show, who found chemical effects by their experiments with ions of an energy range between 4 and $10 \mathrm{keV}$. The process of selective sputtering is strongly depending on the energy of the ions but, probably, also on the experimental conditions (Dukes et al. 1999). A compositional processing of enstatite by interaction of interstellar cosmic rays should only occur at very low energies. Sputtering rates for magnesium silicates have been published by different authors (Wickramasinghe 1972; Falk \& Scalo 1975; Johnson 1994). For magnesium silicate targets maximum sputter yields of 0.01 have been measured for $1 \mathrm{keV} \mathrm{He}{ }^{+}$ions. A complete conversion of olivine in enstatite can only be expected from $\mathrm{He}^{+}$ions in an energy range 
between 1-10 keV. Recent observational results point rather to the maintenance of the chemical composition of silicates in their life cycle (Vriend 2000; Kemper et al. 2001; Bouwman et al. 2001).

Another finding of the present experiments is the formation of pores that confirms analogous results by Demyk et al. (2001). Porous materials have already been formerly proposed in interstellar dust models (Mathis \& Whiffen 1989; Mathis 1996; Demyk et al. 1999), but the porosity was explained mostly by the aggregation of different grain species. We point to other possible mechanisms that could form bubbles in amorphous silicates, e.g. if the grains are formed by vitrification after grain collisions.

The interstellar silicate absorption spectra do not allow any doubts that almost all silicate particles in the diffuse interstellar medium are completely amorphous. This is also true for the silicate cores in the dust grains of the molecular clouds, as the silicate absorption bands in the spectra of deeply embedded massive young stars and protostars (Demyk et al. 1999) show. Obviously, this is not the case in protostellar disks and $\beta$-Pictoris objects, where clear indications for crystalline silicates exist (for review see Dorschner \& Henning 1995; Dorschner 2001).

However, this does not justify the assumption that all interstellar dust materials are completely amorphized. This is confirmed by the presolar grains isolated from primitive meteorites. These crystalline stardust species can be identified with the corresponding well-known minerals diamond, graphite, titanium carbide, silicon carbide, corund, spinel, and hibonite. Laboratory experiments show, that $\mathrm{MgO}$, spinel and corund particles are strongly radiation resistant (Hobbs et al. 1994; Wang et al. 1998) and find therefore their application in fusion reactors and as dosimeter materials.

We do not know how long these stardust species were actually exposed to the diffuse ISM conditions. Concerning SiC, Whittet et al. (1990) have supposed that the lacking spectral evidence of the strong $\mathrm{SiC}$ band, e.g. in the IR spectrum of Galactic Center sources, could be explained by a destruction of these grains in the oxidizing interstellar environment. That means, the crystalline $\mathrm{SiC}$ grains, isolated from meteorites, should have been younger than $4 \times 10^{8}$ years, when they were incorporated in the interplanetary solids of the arising sun. Ott \& Begemann (2000) argued the validity of former published presolar ages $\left(10-130 \times 10^{6}\right.$ a) of Murchison SiC grains. Experiments on the retention of spallogenic ${ }^{21} \mathrm{Ne}$ points to much lower ages. Here, we are apparently confronted with a selection effect. Vapor-phase condensation experiments have shown that in contrast to silicates $\mathrm{SiC}$ grains inherently condense in the crystalline state (Clément 2002). Furthermore, SiC as a semiconducting material can easily recrystallize from the amorphous structure by combination of ion irradiation and annealing (Heera et al. 1995, 1999) leading to a recrystallization of $3 \mathrm{C}-\mathrm{SiC}$ grains (cubic modification).

We hope that this paper will stimulate further experimental studies devoted to those effects that could amorphize silicates in interstellar space.
Acknowledgements. This work has been supported by the Deutsche Forschungsgemeinschaft (DFG grant Do 575/2-3). We gratefully acknowledge the critical comments by A. P. Jones which helped to improve the quality of the paper.

\section{References}

Biermann, P., Langer, N., Seo, E.-S., \& Stanev, T. 2001, A\&A, 369, 269

Bouwman, J., Meeus, G., de Koter, A., et al. 2001, A\&A, 375, 950

Bradley, J. 1994, Science, 265, 925

Cameron, M., \& Papike, J. 1982, in Rev. Mineralogy, Pyroxenes, ed. C. Prewitt (Chelsea: BookCrafters), 7, 5

Chiar, J., Tielens, A., Whittet, D., et al. 2000, ApJ, 537, 749

Clément, D. 2002, Dissertation, Friedrich-Schiller-Universität Jena

Day, K. 1977, MNRAS, 178, 49P

deJong, T., \& Kamijo, F. 1973, A\&A, 25, 363

Demyk, K., Carrez, P., Leroux, H., et al. 2001, A\&A, 368, L38

Demyk, K., Jones, A., Dartois, E., Cox, P., \& d'Hendecourt, L. 1999, A\&A, 349, 267

Dorschner, J. 2001, in Interplanetary Dust, ed. E. Grün, B. Gustafson, S. Dermott, \& H. Fechtig (Berlin: Springer), 727

Dorschner, J., \& Henning, T. 1995, A\&ARv, 6, 271

Draine, B., \& Li, A. 2001, ApJ, 551, 807

Dukes, C., Baragiola, R., \& McFadden, L. 1999, J. Geophys. Res., 104,1865

Duley, W. 2000, MNRAS, 319, 791

Dwek, E., Foster, S., \& Vancura, O. 1996, ApJ, 457, 244

Dwek, E., \& Werner, M. 1981, ApJ, 248, 138

Ellison, D., Drury, L., \& Meyer, J.-P. 1997, ApJ, 487, 197

Fabian, D., Jäger, C., Mutschke, H., Dorschner, J., \& Henning, T. 2000, A\&A, 364, 282

Falk, S., \& Scalo, J. 1975, ApJ, 202, 690

Farquhar, P., Millar, T., \& Herbst, E. 1994, MNRAS, 269, 641

Fields, B., Olive, K., Cassé, M., \& Vangioni-Flam, E. 2001, A\&A, 370,623

Foti, G. 1991, in Proc. of the International School of Physics Enrico Fermi, Course CXI, held in Varenna, 1989 (Amsterdam: NorthHolland Elsevier Science Publishers B.V.), 73

Fowler, W., Reeves, H., \& Silk, J. 1970, ApJ, 162, 49

Hallenbeck, S., Nuth, J., \& Daukantas, P. 1998, Icarus, 131, 198

Hasegawa, T., \& Herbst, E. 1993, MNRAS, 263, 589

Hayakawa, S., Nishimura, S., \& Takayanagi, K. 1961, Publ. Astron. Soc. Japan, 40, 184

Heera, V., Kögler, R., Skorupa, W., \& Stoemenos, J. 1995, Appl. Phys. Lett., 67, 1999

Heera, V., Stoemenos, J., Kögler, R., Voelskow, M., \& Skorupa, W. 1999, J. Appl. Phys., 85, 1378

Hobbs, L., Clinard Jr., F., Zinkle, S., \& Ewing, R. 1994, Nucl. Mater., 216, 291

Itoh, H. 1988, Publ. Astron. Soc. Japan, 40, 263

Jäger, C., Molster, F., Dorschner, J., et al. 1998, A\&A, 339, 904

Jenniskens, P., Baratta, G., Kouchi, A., et al. 1993, A\&A, 273, 583

Johnson, B. 1994, Physics and Chemistry in Space, vol. 19, Energetic charged-particle interactions with atmospheres and surfaces (Springer-Verlag)

Jones, A. 1997, in From Stardust to Planetesimals, ed. Y. Pendleton, \& A. Tielens (San Francisco: Astron. Soc. Pacific), ASP Conf. Ser., 122, 97

Jones, A., Tielens, A., \& Hollenbach, D. 1996, ApJ, 469, 740 
Kemper, F., Waters, L., de Koter, A., \& Tielens, A. 2001, A\&A, 369, 132

Koike, C., Tsuchiyama, A., Shibai, H., et al. 2000, A\&A, 363, 1115

Krätschmer, W., \& Huffman, D. 1979, Ap\&SS, 61, 195

Léger, A., Jura, M., \& Omont, A. 1985, A\&A, 144, 147

Li, A., \& Draine, B. 2001, ApJ, 550, L213

Lingenfelter, R., Ramaty, R., \& Kozlovsky, B. 1998, ApJ, 500, L153

Lutz, D., Feuchtgruber, H., Genzel, R., et al. 1996, A\&A, 315, L269

Maeder, A. 1983, A\&A, 120, 130

Mathis, J. 1996, ApJ, 472, 643

Mathis, J., \& Whiffen, G. 1989, ApJ, 341, 808

Mennella, V., Baratta, G., Colangeli, L., et al. 1997, ApJ, 481, 545

Mewaldt, R., Leske, R., \& Cummings, J. 1996, in Cosmic abundances, ed. S. Holt, \& G. Sonneborn (San Francisco: ASP), ASP Conf. Ser., 99, 381

Meyer, J.-P., Ellison, D., \& Drury, L. 1997, ApJ, 487, 182

Molster, F., Waters, L., \& Tielens, A. 2002a, A\&A, 382, 222

Molster, F., Waters, L., Tielens, A., Koike, C., \& Chihara, H. 2002b, A\&A, 382

Molster, F., Waters, L., Trams, N., et al. 1999, A\&A, 350, 163

Morfill, G., Voelk, H., \& Lee, M. 1976, J. Geophys. Res., 81, 5841

Mori, M. 1997, ApJ, 478, 225

Moskalenko, I., Strong, A., Ormes, J., \& Potgieter, M. 2002, ApJ, 565, 280

Mysen, B., Virgo, D., \& Seifert, F. 1982, Rev. Geophys. Space Phys., 20,353

Naguib, H., \& Kelly, R. 1975, Radiation effects, 25, 1

Nuth III, J., Hallenbeck, S., \& Rietmeijer, F. 1999, in Laboratory Astrophysics and Space Research., ed. P. Ehrenfreund, C. Kraft, H. Kochan, \& V. Pirronello (Dordrecht: Kluwer), 143

Ott, U., \& Begemann, F. 2000, Meteoritics \& Planetary Science, 35, 53
Papike, J., Ryder, G., \& Shearer, C. 1998, in Rev. Mineralogy, vol. 36, Planetary materials, ed. J. Papike (Washington, D.C.: Mineralogocal Society of America), 196

Prantzos, N., Arnould, M., \& Arcoragi, J.-P. 1987, ApJ, 315, 209

Ramaty, R., Kozlovsky, B., \& Lingenfelter, R. 1996, ApJ, 456, 525

Reynolds, S. 1988, in Galactic and Extra-Galactic Radio Astronomy, ed. G. Verschuur, \& K. Kellermann, 2nd ed. (Berlin: Springer), 439

Scott, E., Taylor, G., Newsom, H., et al. 1989, in Asteroids II, ed. R. P. Binzel, T. Gehrels, \& M. S. Matthews (The University of Arizona Press), 701

Spitzer, L. J., \& Tomasko, M. 1968, ApJ, 152, 971

Strazzulla, G., \& Baratta, G. 1982, A\&A, 266, 434

Strazzulla, G., Calcagno, L., \& Foti, G. 1983, MNRAS, 204, 59

Tielens, A., Seab, C., Hollenbach, D., \& McKee, C. 1987, ApJ, 319, L109

Voshchinikov, N., Semenov, D., \& Henning, T. 1999, A\&A, 349, L25

Vriend, W. 2000, Master's Thesis, Kapteijn Institute, Groningen University, The Netherlands

Waelkens, C., Waters, L., de Graauw, M., et al. 1996, A\&A, 315, L245

Wang, L., \& Ewing, R. 1992, in Material Research Society Symp. Proc. 235 (Pittsburgh: MRS), 333

Wang, L., Gong, R., \& Ewing, R. 1994, in Material Research Society Symp. Proc. 316 (Pittsburgh: MRS), 247

Wang, S., Wang, L., Ewing, R., \& Doremus, R. 1998, J. Non-Cryst. Solids, 238, 198

Waters, L., Molster, F., de Jong, T., et al. 1996, A\&A, 315, L361

Whittet, D., Duley, W., \& Martin, P. 1990, MNRAS, 244, 427

Wickramasinghe, N. 1972, MNRAS, 159, 269

Ziegler, J. 1996, SRIM: The stopping power and range of ions in matter (New York: IBM Publications) 